\title{
Automating Antimicrobial Usage Reporting
}

\author{
Andrew Walsh ${ }^{1}$, Cindy Hou $^{2}$, Nikunj Vyas ${ }^{2}$ \\ ${ }^{1}$ Health Monitoring, Pittsburgh, Pennsylvania, United States \\ ${ }^{2}$ Jefferson Health, Cherry Hill, New Jersey, United States \\ Objective
}

To leverage existing healthcare transaction messages to automate the aggregation of antimicrobial usage statistics in a method compatible with submission to the National Healthcare Safety Network (NHSN) Antimicrobial Usage module.

\section{Introduction}

Antimicrobial stewardship is crucial to the ongoing viability of existing therapies. To facilitate this stewardship, NHSN allows hospitals to submit data on their antimicrobial usage and receive feedback on how their usage compares to other facilities [1]. This feedback can be used by hospital personnel to assess whether their antimicrobial policies are consistent with current best practices.

Participation in this program has so far been limited. There are several barriers to participating, including the challenge of mapping local medication information to the NHSN list of antimicrobials, the burden of tabulating the necessary statistics, and the technical requirements of generating appropriate CDA documents for submission. An automated solution that obtained the necessary data from existing HL7 interfaces and generated CDA documents in the correct format could significantly lower some of the barriers to submitting antimicrobial usage information to NHSN.

\section{Methods}

A continuous feed of HL7 ADT and RAS messages was established between a multi-hospital health system and the EpiCenter syndromic surveillance system. Medication administration data elements included time of administration, patient location, and a facility-specific medication identifier. Patient data included time of admissions, transfers and discharges and all relevant patient locations.

Facility medication codes were reconciled to NHSN antimicrobial identifiers via a multi-step, semi-automated process. A medication formulary was provided by the health system mapping their codes to National Drug Code (NDC) identifiers. The RxNorm API [2] was queried to map NDC identifiers to RxNorm identifiers. A second round of RxNorm API queries linked the formulation-specific RxNorm identifiers to related parent RxNorm identifiers for antimicrobials provided by NHSN. The final mapping from facility medication codes to NHSN antimicrobial identifiers was manually reviewed and edited to remove duplicates and to add links by name that were not found automatically.

NHSN requires reporting by administration route, which was provided in most cases by the formulary. In rare cases, that route was not sufficiently specific and had to be refined by looking at the site of administration for individual doses.

Therapy days were calculated as the total number of unique patient identifiers receiving at least one dose of a given antimicrobial; these were totaled per day, per route, and per inpatient location. Days present were calculated as the total number of unique patient identifiers associated with a given inpatient location at any time during each day. Facility-wide inpatient admissions were calculated as the total number of unique patient identifiers associated with an admission to any inpatient location during each day.

\section{Results}

The RxNorm API yielded mapping between 15,472 NDC identifiers and 847 RxNorm codes, covering 86 (96\%) NHSN antimicrobials. An initial merge using the NDC identifiers from the provided formulary yielded 252 matches to NHSN antimicrobials. Manual reconciliation eliminated duplicates to leave 239 unique antimicrobials from the formulary. Since not all NDC identifiers in the formulary could be associated with an RxNorm code, there was the potential for additional antimicrobials to be present but not matched to an NHSN code. The names of the NHSN antimicrobials were used to search the generic and brand names of medications in the formulary, yielding 6 additional antimicrobials with appropriate routes. After these steps of automated and manual reconciliation and excluding formulations administered via nonreportable routes, a total of 216 antimicrobial formulations were identified that can be reported to NHSN. These covered 67 (74\%) NHSN antimicrobials. 
For July 2018, 206,921 medication administration messages were received, including 11,637 administrations of 48 NHSN antimicrobials at 14 NHSN inpatient locations were observed across all four NHSN routes. These represented $7 \%$ of completed administrations. They accounted for 6,909 days of therapy with all antimicrobials via all routes at all locations. Figure 1 shows the time series of days of therapy by NHSN route of administration. A total of $950(0.6 \%)$ administrations had medication code 99999 and could not be identified.

An additional 189,717 ADT messages for 5,420 distinct visits were received. These yielded 13,885 facility-wide days present and a 14,797 location-specific days present summed across all inpatient locations and facilities, from 4,054 facility-wide admissions. Figure 2 shows the time series of facility-wide days present and summed location-specific days present for each facility.

\section{Conclusions}

Reconciling local facility formularies with a national standardized list of antimicrobials can be a complicated task requiring some amount of human intervention. Once completed, however, HL7 messages from existing interface engines can supply sufficient information for calculating the necessary antimicrobial usage statistics to report to NHSN.

\section{Acknowledgement}

Health Monitoring would like to thank the New Jersey Department of Health for financial support of this work.

\section{References}

1. Centers for Disease Control and Prevention [Internet]. Atlanta: National Health Safety Network; 2017 Dec 29. Antimicrobial Use and Resistance (AUR) Module; 2018 Jan [cited 2018 Sep 10]. Available from: https://www.cdc.gov/nhsn/PDFs/pscManual/11pscAURcurrent.pdf

2. Peters LB, Bodenreider O. 2010. RESTful services for accessing RxNorm. AMIA Annu Symp Proc. $2010,983$.

Figure 1: Time Series of Antimicrobial Days of Therapy by Route

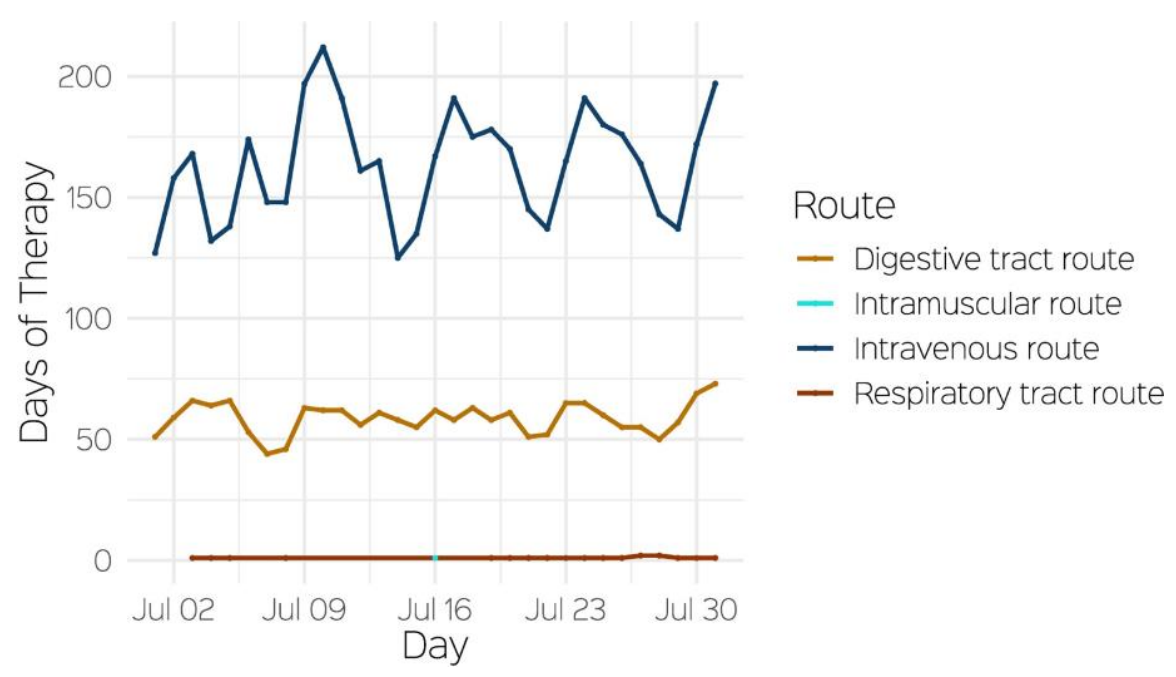

ISDS Annual Conference Proceedings 2019. This is an Open Access article distributed under the terms of the Creative Commons AttributionNoncommercial 4.0 Unported License (http://creativecommons.org/licenses/by-nc/3.0/), permitting all non-commercial use, distribution, and reproduction in any medium, provided the original work is properly cited. 
Figure 2: Time Series of Days-Present from ADT Data by Facility and Aggregation

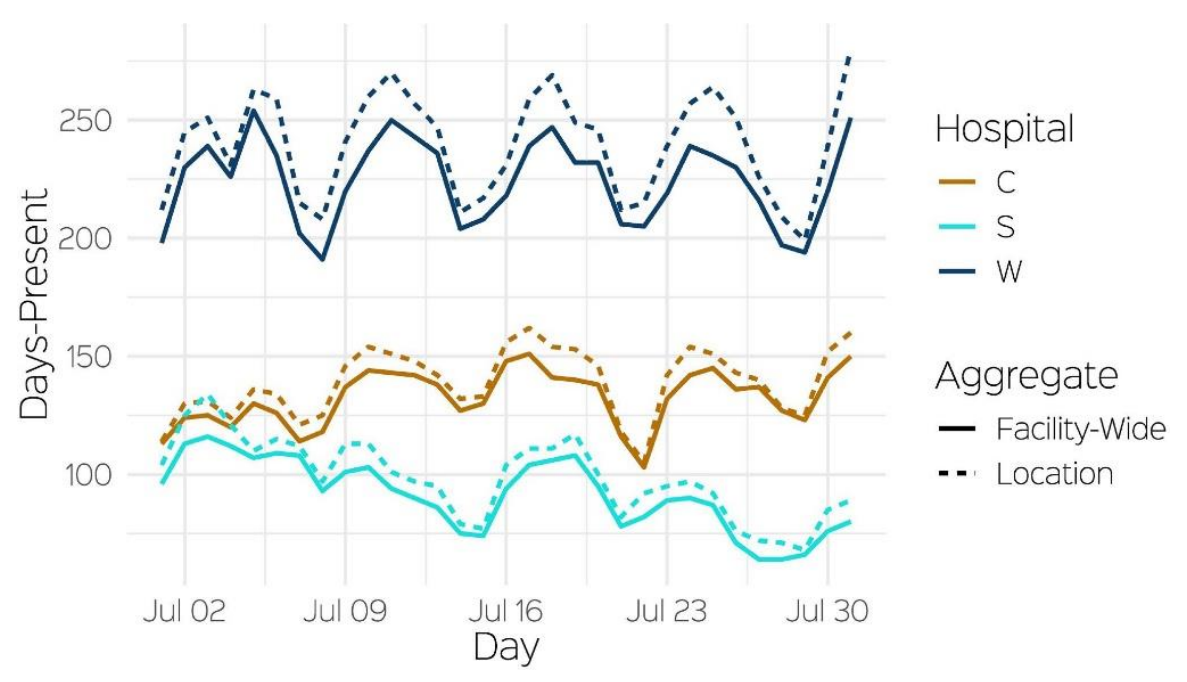

